\title{
Preventing permeability transition pore opening increases mitochondrial maturation, myocyte differentiation and cardiac function in the neonatal mouse heart
}

\author{
Jayson V. Lingan ${ }^{1,2}$, Ryan E. Alanzalon ${ }^{2}$ and George A. Porter Jr2,3,4
}

BACKGROUND: In embryonic myocytes, closure of the mitochondrial permeability transition pore (PTP) drives mitochondrial maturation and cardiac myocyte differentiation. Since neonatal cardiac myocytes remain relatively immature, we hypothesized that inducing PTP closure at this age, by inhibiting the PTP regulator, cyclophilin D (CyPD), genetically or with Cyclosporin A (CSA) and NIM811, would increase cardiac function by increasing mitochondrial maturation and myocyte differentiation.

METHODS: Cultured neonatal myocytes or neonatal mice were treated for $5 \mathrm{~d}$ with vehicle, CsA or NIM811. Mitochondrial function and structure were measured in vitro. Myocyte differentiation was assessed by immunolabeling for contractile proteins. Cardiac function was determined using echocardiography.

RESULTS: The probability of PTP opening was high in WT neonatal myocytes. Treatment with CSA or NIM811 in vitro increased mitochondrial structural complexity and membrane potential, decreased reactive oxygen species levels, and increased myocyte differentiation. WT mice treated with either CSA or NIM811 in vivo for the first $5 \mathrm{~d}$ of life had higher ejection fractions. Deleting CyPD had similar effects as CSA and NIM811 on all parameters.

CONCLUSIONS: It may be feasible to inhibit the PTP using available drugs to increase mitochondrial maturation, myocyte differentiation, and cardiac function in neonates.

N eonatal heart failure remains a significant problem resulting from a wide array of cardiac and noncardiac causes. Occurring with an incidence of $0.87 / 100,000$ or $12,000-35,000$ US children/y, up to $70 \%$ of these patients present in infancy (1). Cardiomyopathies arise from defects in myocyte function, including mitochondrial disease, genetic defects sometimes associated with congenital heart disease, and myocardial stunning after cardiac surgery. Few studies have tested specific therapies for heart failure in infants, and nonspecific therapy is often used, including inotropic and "mitochondrial" reagents (carnitine and coenzyme Q) $(2,3)$.
It has become apparent that mitochondrial maturation and myocyte proliferation/differentiation are linked throughout cardiac development, from the embryo to beyond infancy (4-6). We demonstrated that mitochondria are less active in the early embryonic heart, and as the heart develops, mitochondria attain a more mature structure and function as the electron transport chain (ETC) assembles and activates $(4,5)$. This occurs due to closure of a large conductance channel, the mitochondrial permeability transition pore (PTP), which activates mitochondria, decreases reactive oxygen species (ROS) production, and enhances embryonic myocyte differentiation. Furthermore, inducing PTP closure in embryonic myocytes, using genetic or pharmacologic inhibition of the PTP regulatory protein, cyclophilin $\mathrm{D}(\mathrm{CyPD})$, enhances myocyte differentiation (4).

Birth, perhaps the greatest transition during life, dramatically increases demands on the heart to perfuse the entire organism and profoundly alters the physiologic environment and functional activity of the cardiac myocyte, which responds by undergoing its final stage of differentiation. At birth, the myocardium is immature and neonatal myocytes have a structure that is more "fetal" than "mature" $(6,7)$. The neonatal heart also contains numerous cells that have an early embryonic morphology, making it highly proliferative such that, in the few days of life in the mouse, it can regenerate (8-10). A transition from an embryonic/fetal pattern to a mature pattern of cardiac cellular physiology begins about postnatal day 7 . This contributes to decreased myocyte proliferation, increased differentiation, and a reorganization of myofibrils from a scattered to a highly organized array $(6,7)$.

The bioenergetic signature of the cell changes during this window of final myocyte differentiation $(6,7,11)$. Recent evidence suggests that these changes in bioenergetics and mitochondrial function are required for survival. For example, there is a marked increase in mitochondrial biogenesis, activity, and ultrastructural maturation in the first $2 \mathrm{wk}$ of life that increases mitochondrial ROS production and promotes myocyte differentiation (11). In addition, mitochondrial mitophagy, fusion

\footnotetext{
'Current affiliation: Department of Pediatrics, Benefits Health System, Great Falls, Montana; ${ }^{2}$ Department of Pediatrics, University of Rochester Medical Center, Rochester, New York; ${ }^{3}$ Department of Pharmacology and Physiology, University of Rochester Medical Center, Rochester, New York; ${ }^{4}$ Aab Cardiovascular Research Institute, University of Rochester Medical Center, Rochester, New York. Correspondence: George A. Porter (george_porter@urmc.rochester.edu)
} 
and fission, and metabolically-regulated transcription factors control these changes in bioenergetics and differentiation during the postnatal transition $(8,12)$.

Based on these background data, we sought to determine whether mitochondrial pathways could be manipulated to increase mitochondrial maturation, myocyte differentiation, and cardiac function in the neonatal heart. We hypothesized that neonatal cardiac myocytes remain vulnerable to PTP opening and that preventing PTP opening, or causing its closure, would increase cardiac function by increasing mitochondrial maturation, decreasing ROS generation and enhancing myocyte differentiation, similarly as in the embryonic heart $(4,5)$. We found that inhibiting the PTP in vitro by exposing cultured wild type (WT) and $\mathrm{CyPD}^{-/-}$myocytes to cyclosporin A (CsA), which inhibits CyPD, and NIM811, a nonimmunosuppressive CsA analogue, increased maturation of mitochondrial structure and function, lowered ROS generation, and increased myocyte differentiation but had no effect on proliferation and cell death in vitro. Treatment of 1 day old WT and $\mathrm{CyPD}^{-/-}$mice with CsA and NIM811 also significantly increased cardiac function in neonatal mouse hearts in vivo.

\section{METHODS}

\section{Materials}

Materials were from ThermoFisher/Invitrogen (Waltham, MA) and Sigma (St. Louis, MO) unless noted. NIM811 was from Novartis (Basel, Switzerland).

\section{Animals}

WT C57BL/6N mice were from Charles River Laboratories (Wilmington, MA). CyPD ${ }^{-1-}$ mice in a C57BL/6N background were from the Molkentin Laboratory. All procedures were approved by the University of Rochester Universal Committee on Animal Resources.

\section{Cell Culture}

Ventricles from 4-10 neonatal mice were dissociated with trypsin and collagenase and stopped with soybean trypsin inhibitor (Neonatal Cardiomyocyte Isolation System Kit, Worthington Biochemical Corporation). Samples were plated on gelatin-coated wells or coverslips in Dulbecco's modified Eagle's medium with fetal bovine serum, L-glutamine and antibiotics/antimycotics. Cardiomyocytes were treated daily for $5 \mathrm{~d}$ with vehicle (1:1,000 Ethanol), $500 \mathrm{nmol} / \mathrm{l} \mathrm{CsA}$ or $500 \mathrm{nmol} / 1$ NIM811. Specimens were analyzed live or harvested by fixation at the end of day 5 of treatment (day 6 of culture).

\section{Microscopy}

Primary cultures were labeled with calcein-acetoxymethyl-ester (calcein-AM), 2'-7'-dichlorofluorescin diacetate (DCF), Hoechst 33342, MitoTracker Green (MTG), Sytox orange, and/or tetramethylrhodamine methyl ester (TMRE) (concentrations are listed under individual methods). Live myocytes were identified by their distinct morphology and robust beating. For immunofluorescence labeling, cultures were fixed and labeled, as described (4), with rabbit anti- $\alpha$-actinin (Epitomics (antibody 2310-B)), mouse antitroponin-I (Tn-I) or antitroponin-T (Tn-T; Developmental Studies Hybridoma Bank (TI-4 and RV-C2, respectively)), and/or mouse anti-OSCP (Oligomycin Sensitivity Conferring Protein, Santa Cruz Biotechnology (A8)) then with Alexa 488 or 594 goat-antimouse or goat-antirabbit secondary antibodies. Myocytes were identified by the presence of cardiac-specific $\alpha$-actinin, Tn-I, or Tn-T. Coverslips were mounted with ProLong Gold Antifade Mountant with 4,6-diamidino-2-2-phenylindole (DAPI). Imaging was done using a Zeiss dissecting microscope $(2 \times$ lens) or Zeiss Examiner microscope with LED light sources using $40 \times$ water (N.A. 1.0) or $63 \times$ oil (N.A. 1.4) objectives. We used AxioCam HSm and a Moticam 2,000 cameras. Images were analyzed using Zeiss software (Axiovision, V4.8.2 SP1) and Image J (V1.49, NIH, Bethesda,
MD). Figures were compiled using Photoshop and Illustrator (Adobe, CS3). When fluorescence intensity was measured, identical camera settings and image processing was used.

\section{State of the PTP}

Cultures were exposed to $200 \mathrm{nmol} / \mathrm{l}$ calcein-AM, $20 \mathrm{nmol} / \mathrm{l} \mathrm{TMRE}$, and $500 \mu \mathrm{mol} / \mathrm{l} \mathrm{calcium} \mathrm{cobalt} \mathrm{for} 15 \mathrm{~min}$ at $37^{\circ} \mathrm{C}$, as described (4). Five separate pairs (WT or CyPD) of cultures were analyzed over $2 \mathrm{~d}$, while microscope and camera settings and processing was identical for all analyses. Results are expressed as average minus background fluorescence $\left(\mathrm{F}-\mathrm{F}_{0}\right)$ in areas of interest (AOI) containing mitochondria but not calcein aggregates. Calcein was normalized to TMRE fluorescence, which was not significantly different between samples.

\section{Mitochondrial Structure}

Aspect ratio and form factor were measured in anti-OSCP- labeled mitochondria using Image J (4). Fluorescence images were made binary using a convolve filter, and mitochondrial particles were analyzed for circularity ( $4 \pi$ area/perimeter $\left.{ }^{2}\right)$ and major and minor axes length. Aspect ratio (major axis/minor axis) and form factor (1/circularity) were calculated; both have a minimum of 1 (circular) and are $>1$ with elongation/branching.

\section{Mitochondrial Membrane Potential}

$\Delta \Psi$ was measured using TMRE and MTG, as described (4). Live cultures were loaded for $45 \mathrm{~min}$ at $37^{\circ} \mathrm{C}$ with $20 \mathrm{nmol} / \mathrm{l}$ TMRE and $200 \mathrm{nmol} / \mathrm{l} \mathrm{MTG}$ in (HEPES)-Tyrode's buffer and washed for $5 \mathrm{~min}$ in fresh HEPES-Tyrode's buffer. $\Delta \Psi_{\mathrm{m}}$ is presented as the TMRE/MTG intensity ratio in the AOI.

\section{ROS Levels}

ROS levels were assessed using DCF, as described (4). Live cultures were incubated with $1 \mu \mathrm{mol} / \mathrm{l} \mathrm{DCF}$ for $45 \mathrm{~min}$ at $37^{\circ} \mathrm{C}$ in HEPESTyrode's buffer. Results are expressed as average minus background fluorescence $\left(\mathrm{F}-\mathrm{F}_{0}\right)$ in the AOI.

\section{Live/Dead Cell Assay}

Myocytes were cultured in 12 well plates and analyzed per the manufacturer's instructions and (13). Cultures were incubated in media containing $20 \mu \mathrm{mol} / \mathrm{l}$ calcein-AM, $2.5 \mu \mathrm{mol} / \mathrm{l}$ Sytox orange, and 10 $\mu \mathrm{mol} / \mathrm{l}$ Hoechst for $30 \mathrm{~min}$ at $37^{\circ} \mathrm{C}$. Fluorescence was measured using a Synergy HT Multi-Mode Microplate Reader (BioTek Instruments, Winooski, VT) with absorbance and emission filters set at: Calcein-485/528, Sytox-540/575, and Hoechst-350-360/460. Calcein and Sytox fluorescence were normalized to Hoechst fluorescence.

\section{Myocyte Differentiation}

Cultured myocytes were fixed, permeabilized, and labeled with antibodies to cardiac $\alpha$-actinin, Tn-I, and Tn-T, which identified myocytes. The percentage of myocytes in each culture that had Z- or I-bands was quantified.

\section{Cardiac Function}

One day old WT and CyPD ${ }^{-/-}$mice were not treated or injected intraperitoneal daily for $5 \mathrm{~d}$ with vehicle ( $10 \%$ Solutol in saline), $10 \mathrm{mg} /$ $\mathrm{kg}$ CsA, or $10 \mathrm{mg} / \mathrm{kg}$ NIM811 (14). Hearts were analyzed for cardiac function at postnatal day (PND) 6. Mice were anesthetized with $2 \%$ Isofluorane, and immobilized to a temperature-controlled $\left(37^{\circ} \mathrm{C}\right)$, heated stage. Imaging was performed using a Vevo770 ultrasound machine (40 MHz probe, FujiFilm VisualSonics). Two-dimensional and $\mathrm{M}$-mode images were obtained and analyzed offline using manufacturer's software.

\section{Histology}

On PND 6, mice were sacrificed by cervical dislocation. Hearts were perfusion fixed with $4 \%$ paraformaldehyde and postfixed in neutral buffered formalin. Specimens were embedded in paraffin, and sections were stained with hematoxylin and eosin for imaging. Ventricular dimensions were measured using $2 \times$ images.

\section{Statistics}

Analyses used Prism (V6, GraphPad, La Jolla, CA). Statistical details are presented in Supplementary Table S1 online, and include: two groups-unpaired, two-tailed, Mann-Whitney U-test; multiple groups, normal distribution-one-way or repeated measures 


\section{Articles | Lingan etal.}

ANOVA with Dunnett's or Holm-Sidak's tests; and multiple groups, non-normal distribution-Kruskal-Wallis or Friedman (repeated measures) with Dunn's test. A $P$-value $<0.05$ was considered significant, all experiments were performed at least three times, and data is presented as mean \pm SEM.

\section{RESULTS}

\section{The PTP is Open in Neonatal Cardiac Myocytes}

Based on our previous work (4), we hypothesized that the PTP is more likely to be open in WT neonatal myocytes than in myocytes with a PTP that is closed after CyPD deletion. Therefore, we examined the state of the PTP in cultured ventricular myocytes in media containing calcein-AM and cobalt, which quenches calcein fluorescence. Since both calcein and cobalt can enter the cytoplasm, cytoplasmic calcein will be quenched by cobalt. However, if the PTP is closed, only calcein can enter mitochondria, where it is not quenched. In contrast, with an open PTP, cobalt enters the mitochondrion and quenches calcein. Therefore, if all mitochondria, identified by another form of labeling, such as with TMRE, are labeled with calcein, then the PTP is closed, while if mitochondrial calcein is lower or absent in some mitochondria, then their PTP is open $(4,15)$.

In all live-cell experiments, myocytes were identified by morphology and robust beating. As with early embryonic myocytes (4), cobalt significantly decreased mitochondrial calcein fluorescence in WT vs. CyPD ${ }^{-/-}$neonatal myocytes when calcein labeling was normalized to TMRE labeling $(P=0.001$, Figure 1). Analysis of calcein labeling alone showed a significant difference between the genotypes $(P<0.0001)$. In contrast, TMRE labeling was lower in WT but not significantly different $(P=0.0893)$. This suggests a high probability of PTP opening in mitochondria in early WT neonatal myocytes. In the following sections, we explore the results of preventing this PTP opening in the neonatal heart.

\section{PTP Inhibition Increased Maturation of Mitochondrial Morphology}

Next, we examined mitochondrial morphology in cultured ventricular myocytes. After $5 \mathrm{~d}$ of treatment, mitochondria in WT cultured cardiac myocytes treated with vehicle were more fragmented, round, and dilated compared with myocytes treated with either CsA or NIM811, where mitochondria were elongated and branched, forming thin, filamentous, interconnected networks (Figure 2a). Similar changes were associated with the mitochondrial maturation that occurs with myocyte differentiation in embryonic hearts (4). Furthermore, treatment with CsA and NIM811 expanded the mitochondrial network from around the nucleus to a more linear pattern spanning the length of the cell (Figure 2a). This mature mitochondrial network was also present in $\mathrm{CyPD}^{--}$myocytes, regardless of treatment (Figure 2b). Statistical analysis confirmed this increase in elongation and branching (aspect ratio and form factor) of mitochondria induced by PTP closure in neonatal myocytes (Figure 2c,d).

\section{PTP Inhibition Increased Mitochondrial Function to a More Mature Phenotype}

We then examined mitochondrial function in cultured ventricular myocytes by measuring mitochondrial membrane a

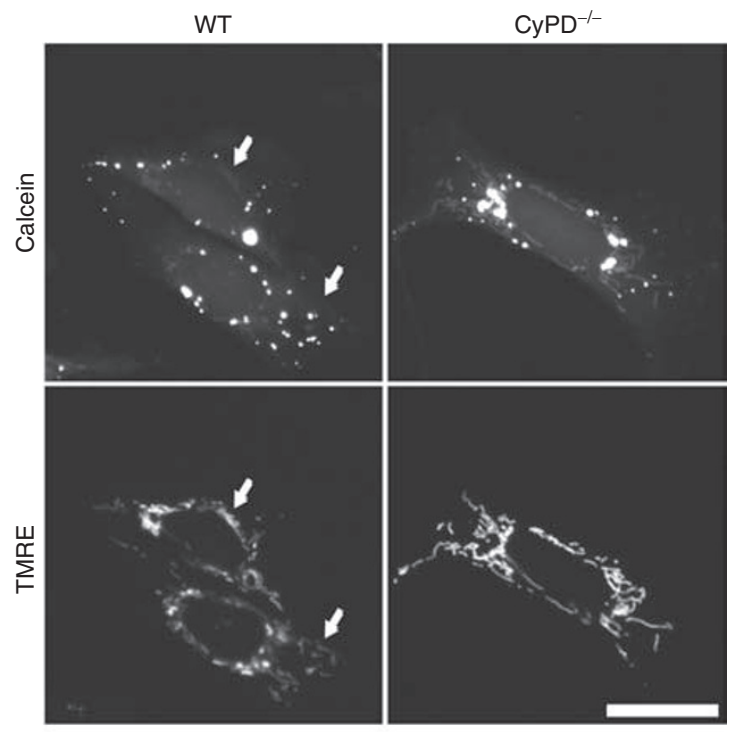

b

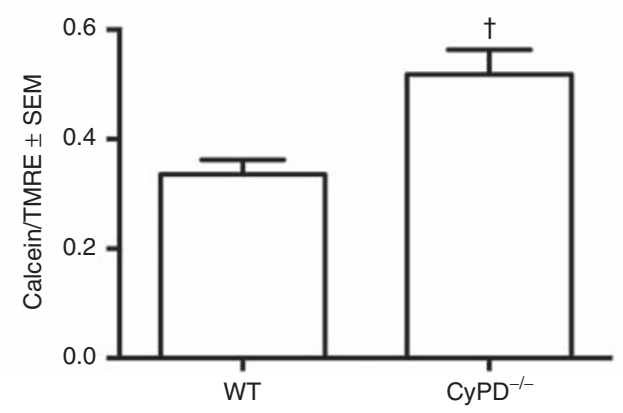

Figure 1. The PTP is open in neonatal cardiac myocytes. One day old primary cultures from neonatal WT and $\mathrm{CyPD}^{-/-}$hearts were incubated with $200 \mathrm{nmol} / \mathrm{I}$ calcein-AM, $20 \mathrm{nmol} / \mathrm{ITMRE}$, and $500 \mu \mathrm{mol} / \mathrm{l}$ cobalt chloride. (a) Photomicrographs of myocytes, identified by morphology and beating, demonstrate typical calcein and TMRE fluorescence. In contrast to CyPD ${ }^{-/-}$cells, not all WT mitochondria (labeled with TMRE) contain calcein (e.g., arrows). Note that intense deposits of calcein were not associated with mitochondria and not included in the analysis. Scale bar $=20 \mu \mathrm{m}$. (b) Quantification of calcein divided by TMRE fluorescence (to normalize for mitochondrial mass/volume) demonstrated significantly lower calcein fluorescence in WT vs. CyPD ${ }^{-/-}$mitochondria. Statistical details in Supplementary Table $\mathbf{S} 1$ online, ${ }^{\dagger} P=0.001$. CyPD, cyclophilin D; PTP, permeability transition pore; TMRE, tetramethylrhodamine methyl ester.

potential $\left(\Delta \Psi_{\mathrm{m}}\right)$ and ROS levels. To compare $\Delta \Psi_{\mathrm{m}}$ between specimens, the ratio was taken of TMRE to MTG intensity, whose retention in the mitochondria is and is not dependent on $\Delta \Psi_{\mathrm{m}}$, respectively (Figure 3a). Statistical analysis revealed that $\Delta \Psi_{\mathrm{m}}$ was higher when WT myocytes were treated with either CsA or NIM811 and when CyPD ${ }^{-/-}$myocytes, regardless of treatment, were examined (Figure $3 \mathbf{b}$ ).

Myocyte ROS levels, assayed with DCF, were high in WT cardiac myocytes treated with vehicle and decreased after treatment with CsA or NIM811 for $5 \mathrm{~d}$ (Figure 3c). A lower DCF fluorescence was also observed in $\mathrm{CyPD}^{-/-}$mice, regardless of treatment (Figure 3c). Quantification confirmed these observations (Figure 3d). These findings indicate that mitochondrial function (elevated $\Delta \Psi_{\mathrm{m}}$ and lower ROS production) matures in coordination with mitochondrial network 
a
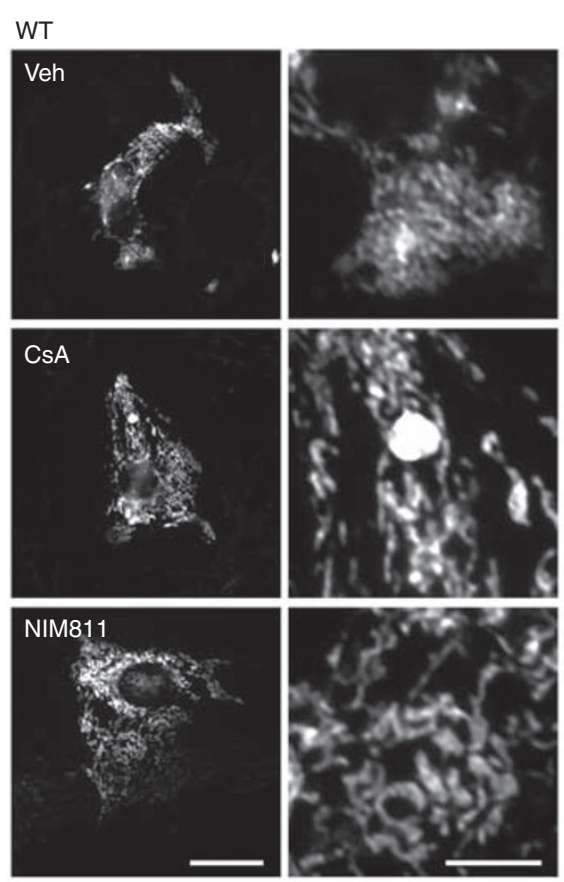

C

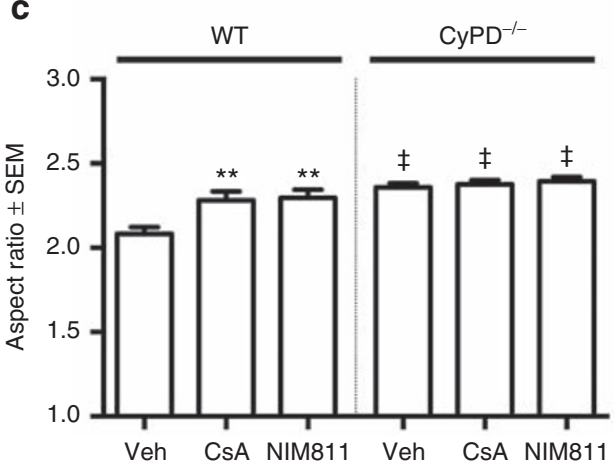

b
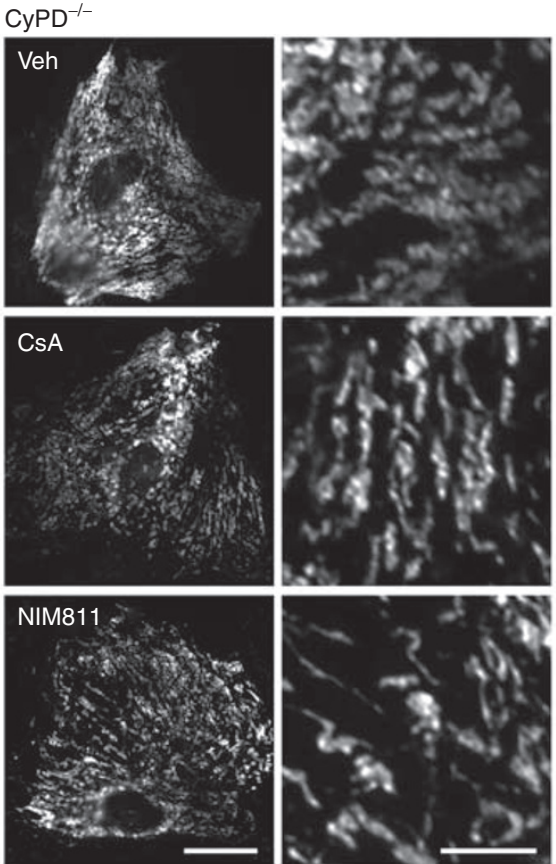

d

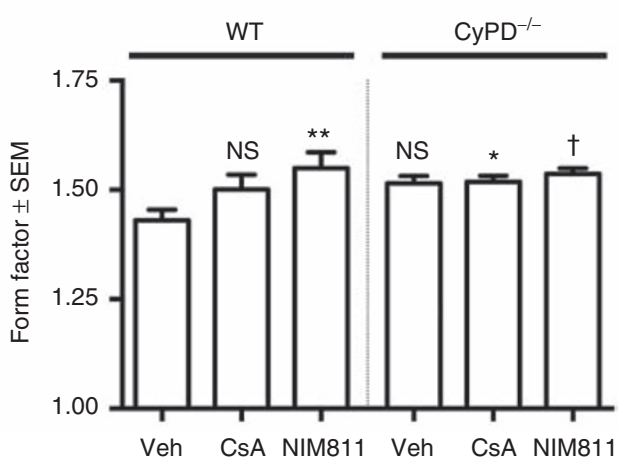

Figure 2. PTP inhibition increased maturation of mitochondrial morphology. Cultured WT and CyPD $\mathrm{PD}^{--}$ventricular myocytes treated with vehicle (Veh), $500 \mathrm{nmol} / \mathrm{I} \mathrm{CsA}$, or $500 \mathrm{nmol} / \mathrm{I} \mathrm{NIM811}$ were stained with anti-OSCP antibody to label mitochondria. (a, b) Low (left, scale bar $=20 \mu \mathrm{m})$ and high (right, scale bar $=5 \mu \mathrm{m}$ ) magnification images of WT (a) and CyPD ${ }^{--}$(b) samples demonstrate a mature mitochondrial morphology when the PTP was closed (treatment with CSA and NIM811, CyPD ${ }^{-/}$) compared with control (Veh, WT). (c, d) Significant changes in aspect ratio (mitochondrial length) and form factor (network complexity) were associated with PTP closure. Statistical details in Supplementary Table S1 online; NS, not significant, ${ }^{*} P<0.05$, ${ }^{* *} P<0.01$, ${ }^{+} P<0.001,{ }^{\ddagger} P<0.0001$. CsA, Cyclosporin A; CyPD, cyclophilin D; OSCP, oligomycin sensitivity conferring protein; PTP, permeability transition pore.

maturation when the PTP is inhibited in cultured neonatal myocytes.

\section{PTP Inhibition Enhanced Neonatal Cardiac Myocyte Differentiation}

To determine if PTP inhibition increases neonatal myocyte differentiation, the proportion of myocytes with Z- or I-band labelling was quantified to assess myofibrillar organization. We previously measured cardiac myocyte differentiation by quantifying the number of $\alpha$-actinin-labeled Z-bands per cell (4), but in this series of experiments, we also labeled for I-bands using anticardiac troponin I (Tn-I) and troponin T ( Tn-T) antibodies. In control cultures, we found an increase in the percentage of cells labeled for both Z- and I-bands over $5 \mathrm{~d}$ in culture (see Supplementary Figure S1 online). When WT myocytes were treated for $5 \mathrm{~d}$ with either CsA or NIM811, there was a significantly higher proportion of cardiac myocytes containing Z- and I-bands (Figure 4a,c,d and Supplementary Figure S2 online). Compared with WT myocytes, cultured CyPD ${ }^{-/-}$ myocytes had larger and more differentiated cardiac myocytes, regardless of treatment (Figure $\mathbf{4 b}-\mathbf{d}$ and Supplementary Figure S2 online). These results suggest that the myocyte contractile apparatus assembles over time in culture and that closure of the PTP accelerates this aspect of differentiation.

To determine if these treatments affected cell survival, WT and $\mathrm{CyPD}^{-/-}$cultures were assessed for live and dead cell numbers. After $5 \mathrm{~d}$ of treatment, there were no differences in the number of live (Figure 4e) or dead (Figure 4f) cells. Therefore, although differentiation was affected by PTP inhibition, cell survival and death were not. 


\section{Articles $\mid$ Lingan et al.}

TMRE/MTG

WT
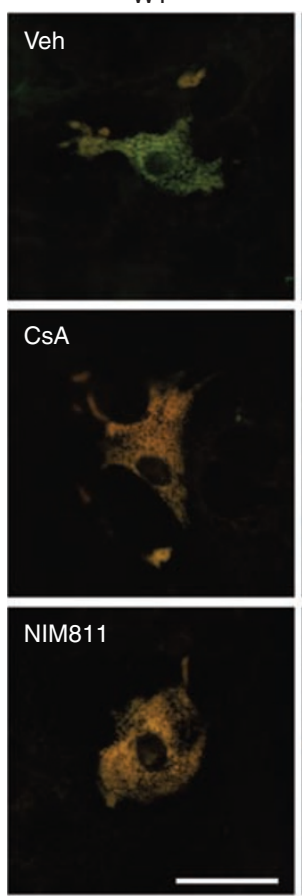

b

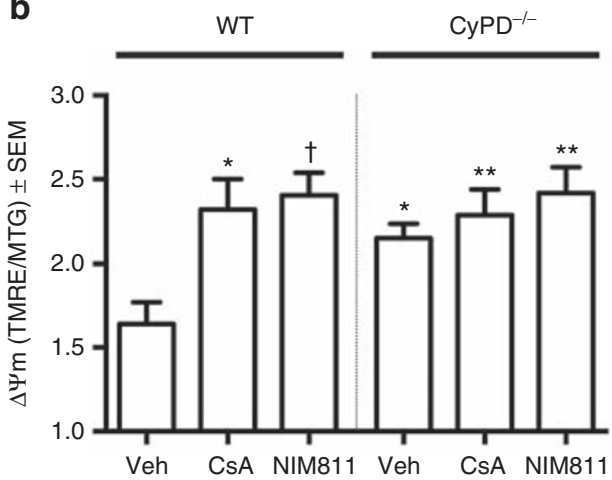

DCF

WT

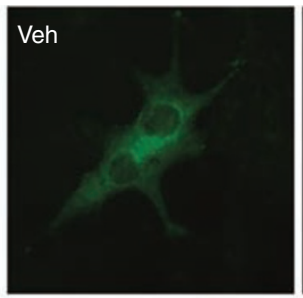

CsA

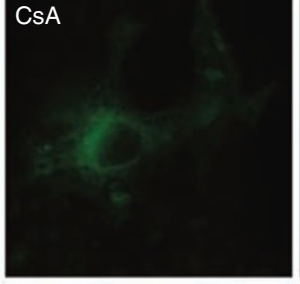

NIM811

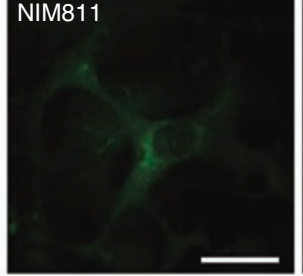

CyPD $^{-/-}$

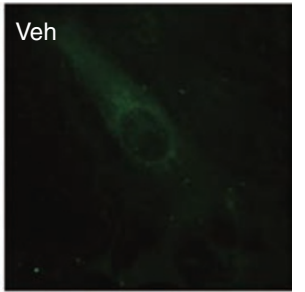

CsA

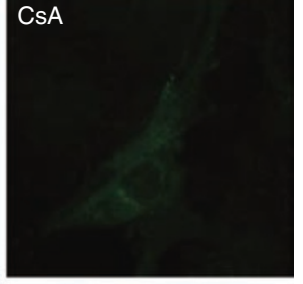

NIM811

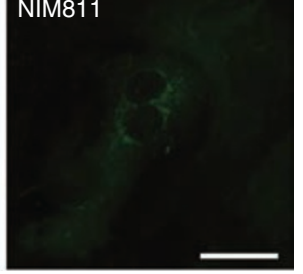

d

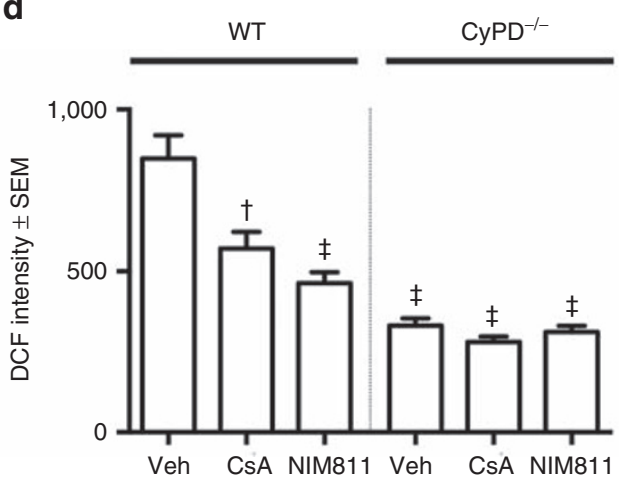

Figure 3. PTP inhibition increased mitochondrial functional maturity. Cultured ventricular myocytes from WT and CyPD ${ }^{-/-}$mice treated with vehicle (Veh), $500 \mathrm{nmol} / \mathrm{I} \mathrm{CSA}$, or $500 \mathrm{nmol} / \mathrm{I} \mathrm{NIM811}$ for $5 \mathrm{~d}$ were stained to analyze $\Delta \Psi_{\mathrm{m}}(\mathbf{a}, \mathbf{b})$ and ROS levels (c, d) on day 6. (a) Merged fluorescence micrographs of mitochondria stained with TMRE/MTG; red/orange indicates high $\Delta \Psi_{\mathrm{m}}^{\mathrm{m}}$ and green indicates low $\Delta \Psi_{\mathrm{m}}$. Scale bars $=40 \mu \mathrm{m}$. (b) Statistical analysis

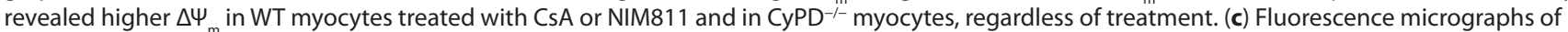
myocytes stained with DCF. Scale bars $=20 \mu \mathrm{m}$. (d) Statistical analysis revealed lower ROS levels (DCF intensity) in WT myocytes treated with either CsA or NIM811 and in CyPD ${ }^{-/-}$myocytes, regardless of treatment. Statistical details in Supplementary Table S1 online; ${ }^{*} P<0.05,{ }^{* *} P<0.01,{ }^{\dagger} P<0.001, \neq P<$ 0.0001. CsA, Cyclosporin A; CyPD, cyclophilin D; DCF, dichlorofluorescin diacetate; MTG, MitoTracker Green; PTP, permeability transition pore; ROS, reactive oxygen species; TMRE, tetramethylrhodamine methyl ester.

\section{PTP Inhibition Increased Cardiac Function in the Neonatal Mouse}

The in vitro experiments presented above suggest that PTP closure in neonatal myocytes accelerates mitochondrial maturation and myocyte differentiation. In the following experiments, we explored whether these changes increase cardiac function in vivo. WT and $\mathrm{CyPD}^{-/-}$neonates were treated with daily intraperitoneal injections of vehicle, CsA and NIM811 from PND 1-5, and on PND 6, parameters of cardiac structure and function were measured using mouse echocardiography.
Control (untreated or vehicle treated) WT neonatal mice had a left ventricular ejection fraction of 65\% (Figure 5a). When the PTP was closed (CsA or NIM811 treatment, CyPD deletion), the ejection fraction increased significantly to 76$80 \%$ (Figure 5a), while fractional shortening increased from $34 \%$ to $41-46 \%$ (Figure $5 \mathbf{b}$ ). In contrast, treatment of $\mathrm{CyPD}{ }^{-/-}$ mice with CsA and NIM811 had no additional effect (Figure 5). Other parameters of left ventricular wall and lumen dimensions were mostly unchanged by these treatments, with only minor differences in left ventricular inner diameter and posterior wall thickness (see Supplementary Figure S3 online). 
a

WT
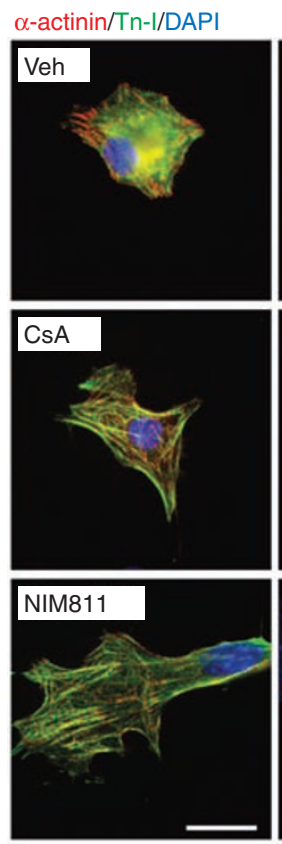

C

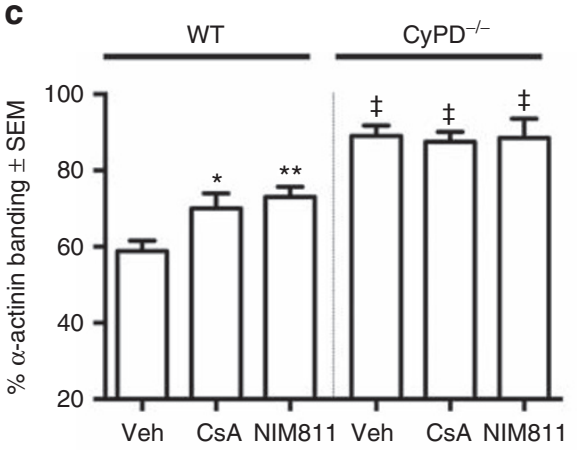

e
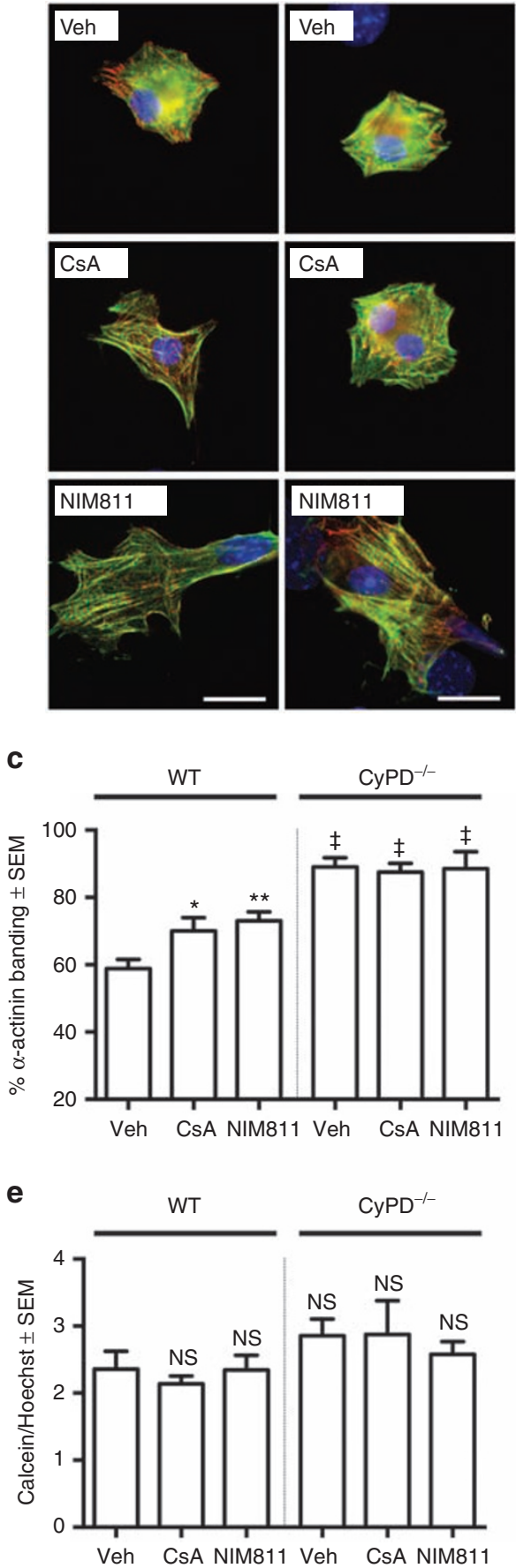

b

CyPD ${ }^{-1-}$

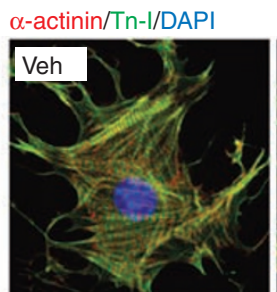

$\alpha$-actinin/Tn-T/DAPI
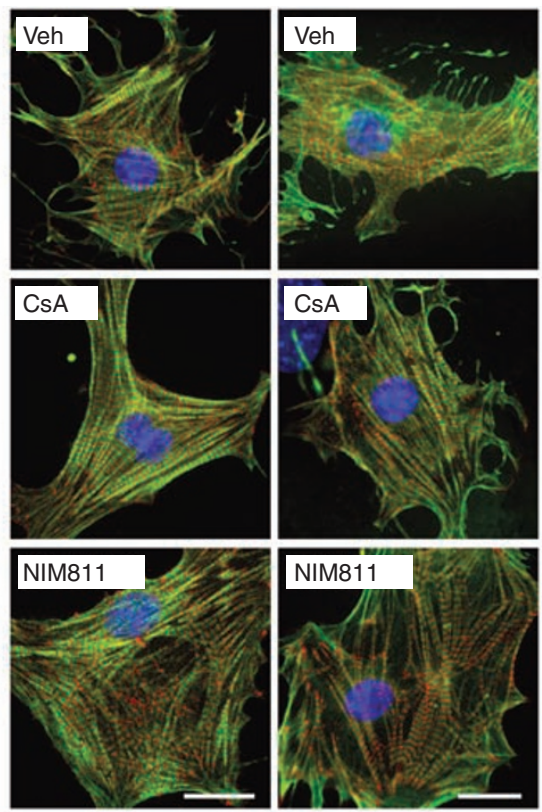

d

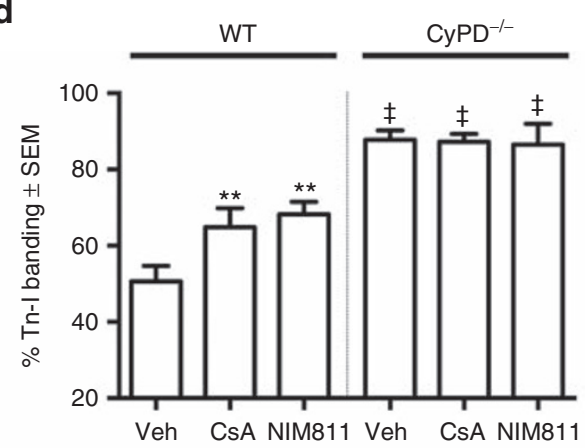

f

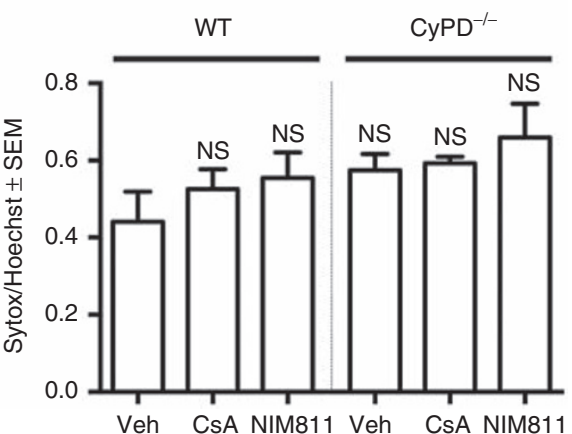

Figure 4. PTP inhibition enhanced neonatal cardiac myocyte differentiation. Cultured ventricular myocytes from WT and CyPD ${ }^{-/-}$mice were treated with vehicle (Veh), $500 \mathrm{nmol} / \mathrm{I} \mathrm{CsA}$, or $500 \mathrm{nmol} / \mathrm{I} \mathrm{NIM811}$ for $5 \mathrm{~d}$ and fixed and labeled one day later. (a, b) Merged fluorescence micrographs of WT (a) and $\mathrm{CyPD}^{-/-}(\mathbf{b})$ myocytes stained for $\alpha$-actinin, DAPI and Tn-I or Tn-T demonstrate increased myocyte size and more organized myofibrils when the PTP is closed (WT with CsA or NIM811, all CyPD ${ }^{-/}$). See Supplementary Figure S2 online for individual channels. Scale bars $=20 \mu \mathrm{m}$. (c, d) Quantification of the percentage of myocytes positive for Z-bands (c, $\alpha$-actinin) and I-bands (d, Tn-I) demonstrate that PTP closure increased myocyte differentiation. (e, $\mathbf{f}$ ) When cultures were labeled with calcein-AM, Sytox orange, and Hoechst, there were no differences in live (e, Calcein/Hoechst) or dead (f, Sytox/Hoechst) cells between treatments. Statistical details in Supplementary Table S1 online; NS, not significant, ${ }^{*} P<0.05,{ }^{* *} P<0.01,{ }^{\ddagger} P<0.0001$. CsA, Cyclosporin A; CyPD, cyclophilin D; DAPI, 4,6-diamidino-2-2-phenylindole; PTP, permeability transition pore; Tn-I, antitroponin-l; Tn-T, antitroponin-t. 


\section{Articles | Lingan et al.}

To determine if these treatments altered gross cardiac morphology, we examined histologic sections. In contrast to measurements made by echocardiography, measurements of chamber and wall diameters in low power histologic images revealed only minor changes in right ventricular wall thickness (Figure 6a,b and Supplementary Figure S4 online).

\section{DISCUSSION}

Maturation of cardiac myocytes during development is essential to meet the functional and metabolic demands of growth $(6,7)$. However, although evidence indicates that mitochondria are important throughout cardiac development $(7,8,11,16)$, the mechanisms, by which these nodes of energy production and intracellular signaling regulate cardiac development, remain poorly understood. We previously showed that early embryonic myocyte differentiation is controlled by mitochondria $(4,5)$. However, we noted a limited clinical applicability of these embryonic studies due to the early stage of development and that PTP opening may be a probabilistic function of development; it is much more likely to be open early in myocyte differentiation.

Here, we provide data suggesting that mitochondria, particularly the PTP, can be manipulated in the neonatal heart and that this may have clinical applicability. We show that the PTP has a high probability of opening in neonatal myocytes (Figure 1), and that closing/inhibiting the PTP in myocytes
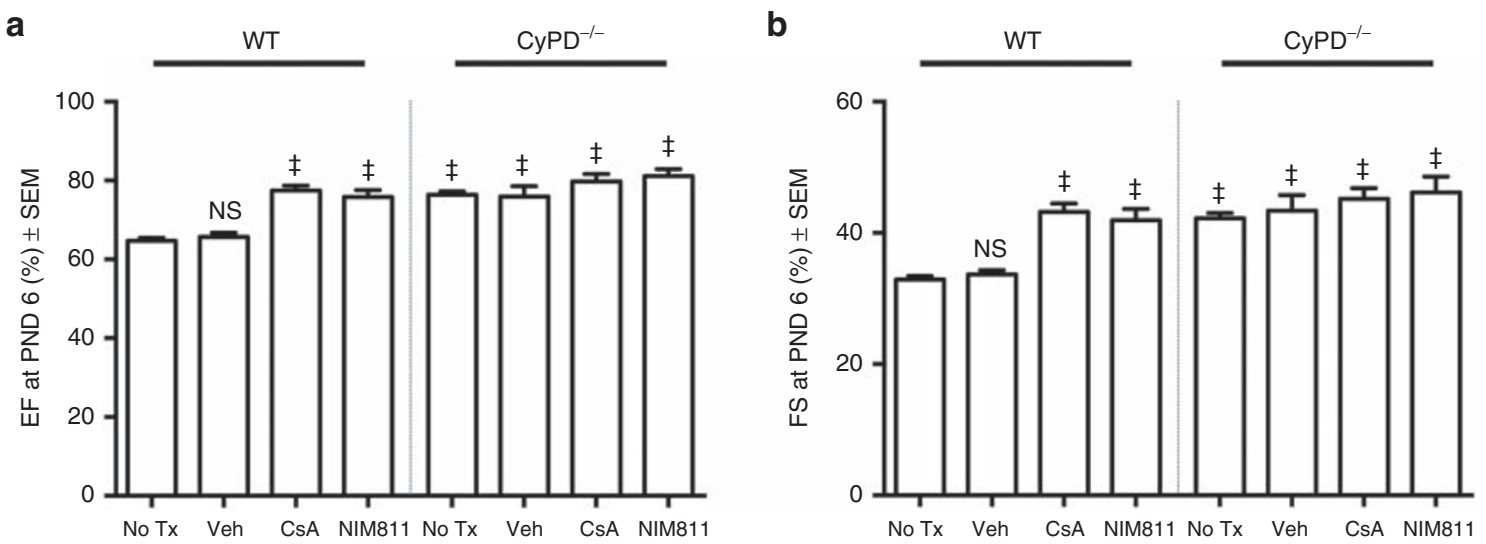

Figure 5. PTP inhibition increased neonatal mouse cardiac function. WT and CyPD ${ }^{-/-}$mice were not treated (No Tx) or treated with daily injections of vehicle (Veh) or CSA or NIM811 for $5 \mathrm{~d}$. Echocardiography performed at PND 6 revealed increased ejection fraction (a, EF) from 65\% to 76-80\% and increased fractional shortening (b, FS) from $34 \%$ to $41-46 \%$ when the PTP was closed (WT + CsA or NIM 811 or CyPD ${ }^{-/-}$). Treatment of CyPD ${ }^{-/-}$mice with CsA and NIM811 had no additional effect. Statistical details in Supplementary Table S1 online; NS, not significant, ${ }^{\ddagger} P<0.0001$. CsA, Cyclosporin A; CyPD, cyclophilin D; PTP, permeability transition pore.
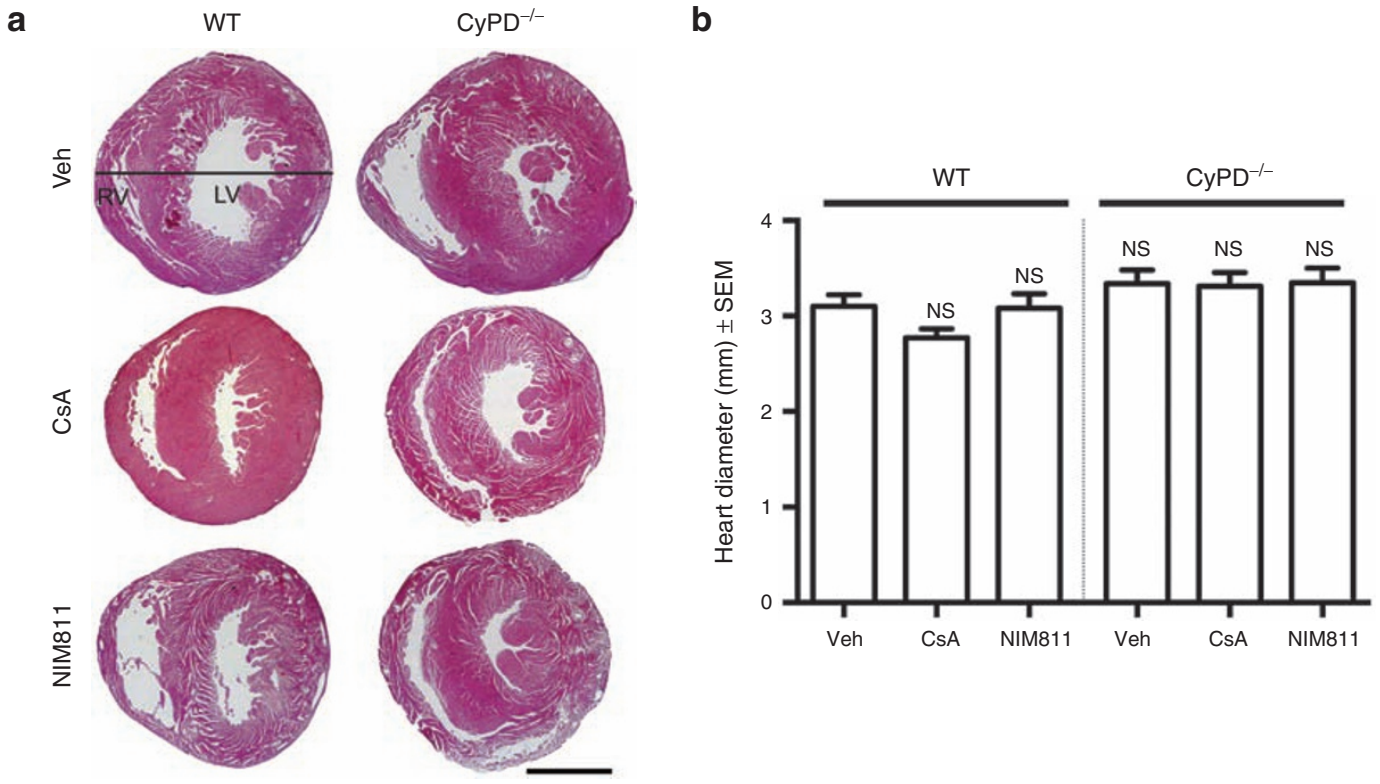

Figure 6. PTP inhibition in vivo did not affect heart size. WT and CyPD ${ }^{-/-}$mice were treated with daily injections of vehicle (Veh) or CsA or NIM811 for $5 \mathrm{~d}$, and hearts were harvested for hematoxylin and eosin staining 1 day after the last treatment. (a) Low power, short axis images of the left and right ventricles (LV, RV) demonstrate no changes in cardiac structure. Scale bar $=1 \mathrm{~mm}$. (b) There were no differences in total heart diameter (black bar in a-WT, Veh) between the groups. Statistical details are in Supplementary Table S1 online; NS, not significant. CsA, Cyclosporin A; CyPD, cyclophilin D; PTP, permeability transition pore. 
at this stage of development with CsA and NIM811 or CyPD deletion increased maturation of mitochondrial morphology (Figure 2) and increased $\Delta \Psi_{\mathrm{m}}$ and decreased ROS production (Figure 3). These changes were associated with increased myocyte differentiation, as indicated by increased contractile apparatus assembly, and did not affect cell survival (Figure 4). Furthermore, in vivo treatment with these agents for the first $5 \mathrm{~d}$ of life significantly increased cardiac function (Figure 5) without significantly changing cardiac structure (Figure 6). Overall, these new data suggest that the PTP is more likely to be open in neonatal myocytes and that closing it or preventing its opening, as in the embryonic heart, enhances mitochondrial maturation, myocyte differentiation, and cardiac function.

\section{Studying the PTP}

Although the permeability transition of mitochondria was discovered in the 1950s, the molecular nature of the PTP has remained a mystery until recently, making studying it difficult. However, the clinically available immunosuppressant, CsA, was found to inhibit its opening, and this led to the identification of CyPD, CsA's target, as the major PTP regulator (17). As CsA also inhibits the cytoplasmic, calcineurin pathway, CsA analogues (e.g., NIM811 and Debio025), which inhibit CyPD at submicromolar concentrations without inhibiting calcineurin, were developed (18). To study the PTP, CsA is generally used at a concentration of $500 \mathrm{nmol} / \mathrm{l}$ or a nonimmunosuppressive CyPD analogue is used.

In clinical studies, CsA and NIM811 protect the heart from ischemia-reperfusion injury (19), but a more recent trial demonstrated that CsA infusion during a myocardial infarction was not protective (20). NIM811 and Debio025 have been used in clinical trials for Hepatitis $\mathrm{C}$, but their use has been curtailed due to other agents' effectiveness and pancreatitis in some patients receiving Debio025 in combination with interferon (21). Further trials using Debio025 are ongoing, but NIM811 is no longer available for use in trials (Novartis, personal communication), and these agents have not been used in neonates.

We recognize that these pharmacologic agents and CyPD deletion affect all cells in the heart and not just myocytes. However, although conditional CyPD deletion has rarely been used and only in other organs, conditional re-expression of CyPD in cardiac myocytes rescued the maladaptive response to transaortic constriction in $\mathrm{CyPD}^{-/-}$mice (22). To address this issue, future experiments can be done using conditional deletion of CyPD.

\section{The PTP in the Neonatal Heart}

To demonstrate the state of the PTP in neonatal myocytes, we used the calcein/cobalt quenching assay. This assay relies on the ability of calcein to enter all cellular compartments, but its fluorescence is quenched by cobalt. Since cobalt entry into mitochondria depends on the PTP being open, if the PTP is closed, then mitochondria will have calcein fluorescence, while, if the PTP is open, they will not. In live cells, this is the only accepted method to specifically determine of the PTP is open $(4,15)$.
When we measured calcein fluorescence normalized to TMRE, values were lower in WT compared with $\mathrm{CyPD}^{-/-}$samples, suggesting that the PTP was more likely to be open in cultured neonatal WT myocytes. This difference is likely due to a combination of two factors: in some mitochondria, the PTP is permanently open, so there is no calcein label, while in others, transient PTP opening decreases, but does not eliminate, calcein fluorescence. These data and that of others, who found that transient opening of the PTP is more common in neonatal than in adult myocytes (23), suggest that a continuous fall in the probability of PTP opening is a property of differentiating myocytes that serves a physiologic purpose (see below). In the future, other methods, such as mitochondrial swelling and calcium retention capacity assays (24), may be used to quantify the susceptibility to PTP opening using isolated mitochondria, while further definition of the molecular identity of the PTP (25-27) may provide additional tools to study PTP activity.

It is possible that the use of cultured cells could affect the state of the PTP and the myocyte, for a number of reasons. First, the dissociation of cells into a monolayer could disrupt cell-cell communication and bioenergetics. Second, exposure to $21 \%$ oxygen, creating a hyperoxic environment with a $\mathrm{pO}_{2}$ of about $150 \mathrm{mmHg}$ (28), might also affect bioenergetics. However, the system of neonatal myocyte culture is well-established to study cardiac cellular physiology, and cultures were performed under identical conditions. Furthermore, we used multiple methods to manipulate one protein, CyPD, who's only apparent function is to regulate the PTP, so PTP activity most likely accounted for observed differences.

\section{PTP Closure Induces Mitochondrial Maturation and Myocyte Differentiation}

To add to our previous work using embryonic myocytes $(4,5)$, we now demonstrate a similar effect in neonatal myocytes: PTP closure increases mitochondrial function and structural maturation, causes a fall in ROS, and induces myocyte differentiation. Other laboratories have demonstrated the importance of mitochondria in regulating balance of proliferation and differentiation in the neonatal heart $(8,11)$. Combined, these data indicate that mitochondrial function regulates myocyte differentiation and function, perhaps by altering the bioenergetic potential of the cell, redox signaling, or other intracellular signaling pathways $(4-8,11)$.

A closed PTP facilitates better coupling of the proximal 4 complexes of the ETC (creates the proton motive force) and ATP synthase (uses the proton motive force) to increase ATP production. We previously showed that closure of the PTP in embryonic hearts is associated with increased ETC assembly and activity $(4,5)$. Herein, we find increased $\Delta \Psi_{\mathrm{m}}$ after PTP closure in neonatal myocytes, suggesting increased ETC efficiency. However, the specific effects of PTP closure on ETC activity and ATP production and how this correlates to postnatal cardiac development and function in vivo will be addressed in future experiments.

Redox signaling may also regulate the balance of proliferation and differentiation during neonatal heart development. 
We previously showed that closure of the PTP in embryonic myocytes decreases ROS levels, thus inducing further myocyte differentiation (4). In these experiments, we find similar results in neonatal myocytes. Other publications also highlight the importance of mitochondrial redox signaling and are beginning to examine the ROS-dependent signaling pathways that control these phenomena $(8,11)$. Redox state and mitochondrial metabolites may also regulate cardiac transcription factors such as MEF2C or GATA4 and hypoxia inducible factor signaling, which are important for cardiac development and regulated by ROS and mitochondrial metabolites (29-31). Finally, the fall in mitochondrial ROS production may be linked to the increased ETC activity discussed above, as ETC assembly decreases ROS production from ETC complexes 1, 2, and $3(5,32)$.

The techniques to study mitochondrial structure and function used in these experiments are standard methods in the field. However, quantifying myocyte differentiation in cultured cells is not so straight-forward. We refined the method used to study embryonic myocytes by using cardiac myocyte-specific antibodies against $\alpha$-actinin, Tn-I, and Tn-T. This not only identified myocytes, but the acquisition of Z- and I-band labeling allowed for quantification of differentiation. We acknowledge that in some live cell experiments, because myocytes were identified by beating, it is possible that we did not examine extremely mature myocytes, which do not beat spontaneously. However, we also identified myocytes based on cellular morphology, and, in our numerous immunolabeling experiments, we did not see any myocytes that resembled isolated mature ventricular myocytes from adult hearts. Furthermore, we used the same identification scheme in all samples to eliminate systematic error across groups.

\section{PTP Closure Increases Cardiac Function in Neonatal Mice}

Most importantly, we found that PTP closure in neonatal mice in vivo significantly increased cardiac function (Figure 5). In vitro experiments suggest that this change in cardiac function is due to increased mitochondrial maturation (Figures 2 and 3 ) and myocyte differentiation (Figure 4). Future experiments will explore the mechanisms that account for the changes in vivo, giving a detailed examination of the state of the PTP and eliminating any artifact introduced by the primary cell culture techniques.

In addition, further delineation of the cell populations affected by these treatments is important. If PTP closure accelerates differentiation of already differentiating myocytes, then this would likely be beneficial in the short-term and not detrimental in the long-term. However, if PTP closure accelerates differentiation of proliferating early myocytes or cardiac stem cells, then this could decrease the total number of myocytes in the mature heart and/or eliminate the ability of the heart to slowly replenish dying myocytes during its lifetime. It is also possible that other cell types, such as fibroblasts, may be affected by PTP closure. Future work can address these issues using additional mouse models (e.g., CyPD conditional deletion) and can also determine whether older neonatal hearts respond to these treatments, neonatal hypoxia affects the window of opportunity for this therapy, and the effects of transient PTP closure in the neonate persist as the animal grows. Furthermore, we might also explore whether these therapies can ameliorate effects of neonatal cardiomyopathies and ischemia; the role of the PTP in the pathophysiology of these diseases is currently unknown.

\section{Summary and Clinical Applicability}

These data demonstrate that neonatal cardiac myocytes are responsive to PTP closure using CsA and NIM811 and deletion of CyPD. PTP inhibition increased mitochondrial maturation and induced myocyte differentiation in vitro. More importantly, closure of the PTP in vivo significantly increased cardiac function in the first week of life. These results suggest that manipulation of mitochondria and the PTP, using clinically available drugs, may be useful to increase cardiac function in neonates with congestive heart failure caused by primary cardiomyopathies, cyanotic or other heart defects, or cardiac bypass. Furthermore, because these drugs would cause transient closure of the PTP during this neonatal window, then the potential detrimental effects of permanent PTP closure seen in the $\mathrm{CyPD}^{-/-}$mice (22) might be avoided.

\section{SUPPLEMENTARY MATERIAL}

Supplementary material is linked to the online version of the paper at http://www.nature.com/pr

\section{ACKNOWLEDGMENTS}

We thank Gisela Beutner, Paul Brookes, Michael O'Reilly, Yves Wang and Dr. Lingan's Scholarly Oversight Committee for helpful discussions, Julianne Feola and Gail Johnson for help with live/dead cell assays, and Novartis for providing NIM811.

\section{STATEMENT OF FINANCIAL SUPPORT}

This work was supported by grants from the American Heart Association Founder's Affiliate (12GRNT12060233), the Strong Children's Research Center at the University of Rochester, and the Matthew's Hearts of Hope Foundation.

Disclosure: The authors have no potential/perceived conflicts of interests to disclose.

\section{REFERENCES}

1. Hsu DT, Pearson GD. Heart failure in children: part I: history, etiology, and pathophysiology. Circ Heart Fail 2009;2:63-70.

2. Hsu DT, Pearson GD. Heart failure in children: part II: diagnosis, treatment, and future directions. Circ Heart Fail 2009;2:490-8.

3. Sole MJ, Jeejeebhoy KN. Conditioned nutritional requirements: therapeutic relevance to heart failure. Herz 2002;27:174-8.

4. Hom JR, Quintanilla RA, Hoffman DL, et al. The permeability transition pore controls cardiac mitochondrial maturation and myocyte differentiation. Dev Cell 2011;21:469-78.

5. Beutner G, Eliseev RA, Porter GA Jr. Initiation of electron transport chain activity in the embryonic heart coincides with the activation of mitochondrial complex 1 and the formation of supercomplexes. PLoS One 2014;9:e113330.

6. Baker CN, Ebert SN. Development of aerobic metabolism in utero: requirement for mitochondrial function during embryonic and fetal periods. OA Biotechnology 2013;2:16.

7. Porter GA Jr, Hom J, Hoffman D, Quintanilla R, de Mesy Bentley K, Sheu SS. Bioenergetics, mitochondria, and cardiac myocyte differentiation. Prog Pediatr Cardiol 2011;31:75-81. 
8. Gong G, Song M, Csordas G, Kelly DP, Matkovich SJ, Dorn GW 2nd. Parkin-mediated mitophagy directs perinatal cardiac metabolic maturation in mice. Science 2015;350:aad2459.

9. Zaruba MM, Soonpaa M, Reuter S, Field LJ. Cardiomyogenic potential of C-kit(+)-expressing cells derived from neonatal and adult mouse hearts. Circulation 2010;121:1992-2000.

10. Porrello ER, Mahmoud AI, Simpson E, et al. Transient regenerative potential of the neonatal mouse heart. Science 2011;331:1078-80.

11. Puente BN, Kimura W, Muralidhar SA, et al. The oxygen-rich postnatal environment induces cardiomyocyte cell-cycle arrest through DNA damage response. Cell 2014;157:565-79.

12. Martin OJ, Lai L, Soundarapandian MM, et al. A role for peroxisome proliferator-activated receptor $\gamma$ coactivator- 1 in the control of mitochondrial dynamics during postnatal cardiac growth. Circ Res 2014;114:626-36.

13. Gundemir S, Colak G, Feola J, Blouin R, Johnson GV. Transglutaminase 2 facilitates or ameliorates HIF signaling and ischemic cell death depending on its conformation and localization. Biochim Biophys Acta 2013;1833:110 .

14. Argaud L, Loufouat J, Gateau-Roesch O, Gomez L, Robert D, Ovize M. Persistent inhibition of mitochondrial permeability transition by preconditioning during the first hours of reperfusion. Shock 2008;30:552-6.

15. Petronilli V, Miotto G, Canton M, et al. Transient and long-lasting openings of the mitochondrial permeability transition pore can be monitored directly in intact cells by changes in mitochondrial calcein fluorescence. Biophys J 1999;76:725-34.

16. Folmes CD, Dzeja PP, Nelson TJ, Terzic A. Mitochondria in control of cell fate. Circ Res 2012;110:526-9.

17. Szabo I, Zoratti M. Mitochondrial channels: ion fluxes and more. Physiol Rev 2014;94:519-608.

18. Waldmeier PC, Feldtrauer JJ, Qian T, Lemasters JJ. Inhibition of the mitochondrial permeability transition by the nonimmunosuppressive cyclosporin derivative NIM811. Mol Pharmacol 2002;62:22-9.

19. Argaud L, Gateau-Roesch O, Muntean D, et al. Specific inhibition of the mitochondrial permeability transition prevents lethal reperfusion injury. J Mol Cell Cardiol 2005;38:367-74.
20. Cung TT, Morel O, Cayla G, et al. Cyclosporine before PCI in patients with acute myocardial infarction. N Engl J Med 2015;373:1021-31.

21. Pawlotsky JM, Flisiak R, Sarin SK, et al.; VITAL-1 study team. Alisporivir plus ribavirin, interferon free or in combination with pegylated interferon, for hepatitis C virus genotype 2 or 3 infection. Hepatology 2015;62:101323.

22. Elrod JW, Wong R, Mishra S, et al. Cyclophilin D controls mitochondrial pore-dependent $\mathrm{Ca}(2+)$ exchange, metabolic flexibility, and propensity for heart failure in mice. J Clin Invest 2010;120:3680-7.

23. Wang W, Fang H, Groom L, et al. Superoxide flashes in single mitochondria. Cell 2008;134:279-90.

24. Sokolova N, Pan S, Provazza S, et al. ADP protects cardiac mitochondria under severe oxidative stress. PLoS One 2013;8:e83214.

25. Alavian KN, Beutner G, Lazrove E, et al. An uncoupling channel within the c-subunit ring of the F1FO ATP synthase is the mitochondrial permeability transition pore. Proc Natl Acad Sci USA 2014;111:10580-5.

26. Izzo V, Bravo-San Pedro JM, Sica V, Kroemer G, Galluzzi L. Mitochondrial permeability transition: new findings and persisting uncertainties. Trends Cell Biol 2016;26:655-67.

27. Mnatsakanyan N, Beutner G, Porter GA, Alavian KN, Jonas EA. Physiological roles of the mitochondrial permeability transition pore. J Bioenerg Biomembr 2017;49:13-25.

28. Simon MC, Keith B. The role of oxygen availability in embryonic development and stem cell function. Nat Rev Mol Cell Biol 2008;9:285-96.

29. Buggisch M, Ateghang B, Ruhe C, et al. Stimulation of ES-cell-derived cardiomyogenesis and neonatal cardiac cell proliferation by reactive oxygen species and NADPH oxidase. J Cell Sci 2007;120(Pt 5):885-94.

30. Schmelter M, Ateghang B, Helmig S, Wartenberg M, Sauer H. Embryonic stem cells utilize reactive oxygen species as transducers of mechanical strain-induced cardiovascular differentiation. FASEB J 2006;20:1182-4.

31. Semenza GL. Hypoxia-inducible factors in physiology and medicine. Cell 2012;148:399-408.

32. Lenaz G, Tioli G, Falasca AI, Genova ML. Complex I function in mitochondrial supercomplexes. Biochim Biophys Acta 2016;1857: 991-1000. 